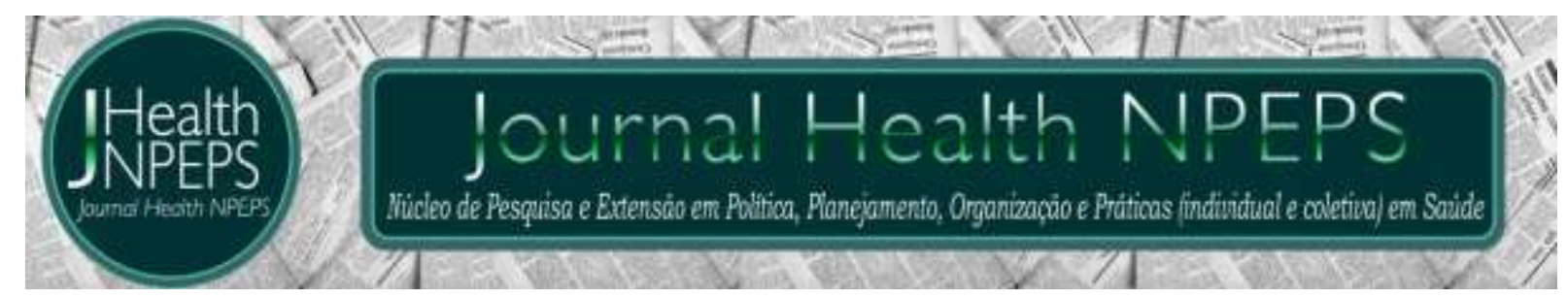

http://dx.doi.org/10.30681/252610103710

ARTIGO ORIGINAL

\title{
Perfil de morbimortalidade da unidade de terapia intensiva de um hospital
} universitário

\section{Profile of morbimortality of the intensive care unit of a university hospital \\ Perfil de morbimortalidad de la unidad de terapia intensiva de un hospital universitário}

\author{
Yasmin Viegas da Cruz ${ }^{1}$, Joana Darc Chaves Cardoso², \\ Carla Rafaela Teixeira Cunha ${ }^{3}$, Akeisa Dieli Ribeiro Dalla Vechia ${ }^{4}$
}

\section{RESUMO}

Objetivo: analisar o perfil de morbimortalidade da unidade de terapia intensiva de um hospital universitário. Método: trata-se de estudo quantitativo e documental, com informações obtidas por meio de prontuários de 86 pacientes internados neste setor hospitalar. Os dados foram coletados a partir de um formulário e organizados em planilhas do programa Microsoft Excel 2013. A análise foi descritiva no formato de tabelas e gráficos, estratificadas por sexo, com frequência absoluta e relativa e as variáveis contínuas em médias. Resultados: identificou-se que a maioria dos usuários internados era mulher, com faixa etária de 39 a 59 anos, casada ou residia com o cônjuge e apresentava doença crônica. As principais causas de internação foram por complicações do pós-operatório, choque séptico e insuficiência respiratória. Mulheres e homens tiveram como causas de mortes mais frequentes pneumonia, disfunção de múltiplos órgãos e choque séptico. Conclusão: o conhecimento do perfil de morbimortalidade dos usuários internados é fundamental para o planejamento e melhoria do cuidado, uma vez que reflete as necessidades e demandas da clientela.

Descritores: Unidades de Terapia Intensiva; Indicadores de Morbimortalidade; Cuidados de Enfermagem; Perfil de Saúde; Epidemiologia.

\section{ABSTRACT}

Purpose: to analyze the morbidity and mortality profile of the intensive care unit of a university hospital. Method: this is a quantitative and documentary study, with information obtained from medical records of 86 patients admitted to this hospital sector. Data were collected from a form and organized into Microsoft Excel 2013 spreadsheets. The analysis was descriptive in the form of tables and graphs, stratified by sex, with absolute and relative frequency and continuous variables as means. Results: it was found that the majority of hospitalized users were women, aged 39 to 59 years old, married or residing with their spouse and presented chronic

\footnotetext{
${ }^{1}$ Enfermeira. Especialista em Saúde do Adulto e do Idoso com Ênfase em Atenção Cardiovascular. Cuiabá, Mato Grosso (MT), Brasil. E-mail: viegascruz8@gmail.com ORCID ID: https://orcid.org/0000-0001-7408-3249 Autor principal - Endereço para correspondência: Avenida Fernando Correa da Costa, 2367, Bairro Boa esperança, Cuiabá $-\mathrm{MT}$, Brasil.

${ }^{2}$ Enfermeira. Mestre em enfermagem. Docente da Faculdade de enfermagem da Universidade Federal de Mato Grosso (UFMT). Cuiabá, Mato Grosso, Brasil. E-mail: joana-qtal@hotmail.com ORCID ID: https://orcid.org/00000003-1989-4043

${ }^{3}$ Enfermeira. Mestre em enfermagem. Docente da Faculdade de enfermagem da Universidade Federal de Mato Grosso (UFMT). Cuiabá, Mato Grosso, Brasil. E-mail: ca rafa enf@hotmail.com ORCID ID: https://orcid.org/00000001-7084-221X

${ }^{4}$ Enfermeira. Mestre em enfermagem. Docente da Universidade do Estado de Mato Grosso (UNEMAT). Cuiabá, Mato Grosso, Brasil. E-mail: akeisa drdv@hotmail.com ORCID ID: https://orcid.org/0000-0002-2749-9695
} meio, desde que a publicação original seja corretamente citada. 
disease. The main causes of hospitalization were postoperative complications, septic shock and respiratory failure. Women and men had the most frequent causes of death as pneumonia, multiple organ dysfunction, and septic shock. Conclusion: knowledge of the morbidity and mortality profile of hospitalized users is fundamental for the planning and improvement of care, as it reflects the customer needs and demands.

Descriptors: Intensive Care Units; Indicators of Morbidity and Mortality; Nursing Care; Health Profile; Epidemiology.

\section{RESUMEN}

Objetivo: analizar el perfil de morbilidad y mortalidad de la unidad de cuidados intensivos de un hospital universitario. Método: Este es un estudio cuantitativo y documental, con información obtenida de registros médicos de 86 pacientes ingresados en este sector hospitalario. Los datos se recopilaron de un formulario y se organizaron en hojas de cálculo de Microsoft.Excel 2013. El análisis fue descriptivo en forma de tablas y gráficos, estratificados por género, con frecuencia absoluta y relativa y variables continuas como medias. Resultados: se encontró que la mayoría de los usuarios hospitalizados eran mujeres, de 39 a 59 años de edad, casadas o que residían con su cónyuge y presentaban enfermedades crónicas. Las principales causas de hospitalización fueron complicaciones postoperatorias, shock séptico $e$ insuficiencia respiratoria. Las mujeres y los hombres tuvieron las causas más frecuentes de muerte como neumonía, disfunción orgánica múltiple y shock séptico. Conclusión: el conocimiento del perfil de morbilidad y mortalidad de los usuarios hospitalizados es fundamental para la planificación y mejora de lo cuidado, ya que refleja las necesidades y demandas del cliente.

Descriptores: Unidades de Cuidados Intensivos; Indicadores de Morbimortalidad; Atención de Enfermería; Perfil de Salud; Epidemiología.

\section{INTRODUÇÃO}

Sabe-se que o Brasil passou por uma intensa transição demográfica e epidemiológica a partir do século $\mathrm{XX}$ com mudanças no perfil de adoecimento, destacando-se as doenças crônico-degenerativas ou doenças crônicas não transmissíveis (DCNT) ${ }^{1}$.

As DCNT são as principais causas de mortes no mundo e têm gerado elevados números de óbitos prematuros. Na Europa, as doenças cardiovasculares (DCV) se destacam como a principal causa de morbidade, sendo responsáveis por mais de $45 \%$ de todas as mortes ao ano ${ }^{2}$. No Brasil, as DCNT também se constituem como um problema de saúde preocupante e, sua ocorrência pode estar relacionada ao processo de envelhecimento da população. Essas doenças são responsáveis por $72,0 \%$ das causas de mortes, com destaque para doenças do aparelho circulatório $(30,0 \%)^{3}$.

Dentre as DCNT, as DCV se constituem como morbidades mais frequentes na população adulta e idosa, podendo levar a várias complicações, por exemplo, internação e morte. 0 estado de Mato Grosso enfrenta grande impacto provocado pelas DCNT e dentre essas, destacam-se as doenças isquêmicas do coração (DIC) e as doenças cerebrovasculares (DCBV) ${ }^{4}$.

Estudo realizado em Ribeirão Preto (SP), com 479 pacientes usuários do Sistema Único de Saúde (SUS), identificou que as doenças do aparelho circulatório representaram $13,0 \%$ das causas de internação, o que acarreta um tempo médio de internação de 10 dias $^{5}$.

Para atender a esse perfil epidemiológico, os hospitais 
adequaram-se, investindo em tecnologias em saúde e profissionais especializados, principalmente nas Unidades de Terapia Intensiva (UTI). Esses investimentos consomem grande parte dos recursos destinados à saúde ${ }^{6}$.

A UTI é um setor hospitalar onde se realizam procedimentos invasivos e de alta complexidade a partir do uso de tecnologias avançadas, equipamentos de ponta e equipe multidisciplinar qualificada ${ }^{7}$. Neste ambiente, assim como em outros setores do hospital, as DCV também prevalecem como principal causa de internação ${ }^{6,7}$.

$\mathrm{Na}$ literatura são encontrados diversos estudos realizados nas UTI. Porém, são escassas investigações que exploram o perfil de morbimortalidade dos adultos e idosos neste setor, principalmente na região Centro-Oeste do país. Conhecer o perfil epidemiológico de uma determinada população é fundamental para o planejamento, organização e operacionalização dos serviços de saúde, bem como para a normatização de atividades técnicas correlatas ${ }^{7}$.

Diante desse cenário questionase: Quais as características sociodemográficas, de condições de saúde e utilização de medicamentos de pessoas internadas na UTI? Quais as características de internação e principais causas de morte de usuários internados na UTI?

Dessa maneira, objetivou-se analisar o perfil de morbimortalidade da unidade de terapia intensiva de um hospital universitário. Acredita-se que essas informações permitirão identificar as necessidades de saúde dessas pessoas, além de serem essenciais aos gestores, aos profissionais de saúde, principalmente os enfermeiros, no planejamento e implementação de uma assistência qualificada, diminuindo a morbimortalidade e complicações das DCV.

\section{MÉTODO}

Trata-se de estudo documental, retrospectivo e quantitativo, realizado na UTI adulto do Hospital Universitário Júlio Müller, em Cuiabá, Mato Grosso (MT), Brasil. O hospital possui 92 leitos, sendo dez leitos de UTI adulto.

Foram selecionados os prontuários dos usuários que estiveram internados na UTI adulto, no período de 01 de junho de 2014 a 01 de junho de 2015. Após a seleção, os prontuários foram lidos minuciosamente visando extrair as informações pertinentes. Coletaram-se os dados de 86 prontuários, nos meses de outubro de 2015 a janeiro de 2016, por meio de um formulário previamente construído, contendo informações sobre as características sociodemográficas, de saúde, da internação hospitalar e de mortalidade.

Foi realizado teste piloto com dez prontuários de usuários com características semelhantes aos que fizeram parte do estudo, visando avaliar o formulário de coleta de dados e identificar as dificuldades/facilidades que seriam vivenciadas.

As variáveis do estudo foram: 
- Características sociodemográficas: faixa etária (16 a 38 anos/ 39 a 59 anos/ 60 anos ou mais), sexo (masculino/feminino); estado conjugal (solteiro (a) /casado (a) união-estável, mora junto com companheiro (a) / viúvo (a)).

- Condições de saúde: Problema de saúde crônico (sim/não); principais problemas de saúde (doenças cardiovasculares/ doenças do aparelho respiratório/ neoplasias/doenças hepáticas/ doenças do aparelho excretor/doenças do sistema gastrointestinal/ síndrome da imunodeficiência adquirida/ outros); principais medicamentos utilizados (anti-hipertensivos/ diuréticos/ hipoglicemiantes/ antieméticos e antiulcerosos/_anticonvulsivantes/ corticoides/ antiplaquetários/ antiinflamatórios/ outros).

- Características da internação na UTI: unidade de procedência (unidades do próprio hospital/ hospital público/ hospital privado), principais causas de internação (pós-operatório/ insuficiência respiratória/ choque séptico/ problemas relacionados à gravidez e puerpério/ emergência cardiovascular/ hemorragias/ insuficiência renal/ outros), tempo de internação (dias) (até 10 dias/ 11 a 20 dias/ 21 a 30 dias/ 31 dias ou mais), média de internação e desfecho clínico (alta da UTI / óbito).

- Causas dos óbitos: Principais causas de morte (Choque séptico/ Disfunção de múltiplos órgãos/ Pneumonias/ Insuficiência renal/ Neoplasias/ Doença Pulmonar Obstrutiva Crônica (DPOC)/
Insuficiência respiratória/ Síndrome da Imunodeficiência Adquirida/ Diabetes Mellitus/ Choque hipovolêmico/ Acidente Vascular Cerebral (AVC)/ Outros).

Os dados foram organizados em planilha no Microsoft Excel 2013 e as variáveis foram analisadas de forma descritiva, estratificadas por sexo. As variáveis categóricas foram apresentadas em frequências absolutas e relativas e as variáveis contínuas em médias, dispostas em tabelas e gráficos.

Esta pesquisa foi aprovada pelo Comitê de Ética em Pesquisa do Hospital Universitário Júlio Müller sob parecer número 1.084.012 e CAAE: 43649615.4.0000.5541. Foram seguidos todos os requisitos da Resolução $\mathrm{N}^{\circ}$ 466/12 Conselho Nacional de Saúde (CNS).

\section{RESULTADOS}

A maioria das pessoas internadas na UTI era mulher $(56,9 \%)$, faixa etária de 39 a 59 anos $(36,1 \%)$, casada ou tinha companheiro (53,4\%)(Tabela 1).

Tabela 1 - Distribuição do número e percentual dos usuários internados na UTI, segundo características sociodemográficas. Cuiabá -MT, Brasil. 2016.

\begin{tabular}{|c|c|c|}
\hline Variável & $\mathbf{n}$ & $\%$ \\
\hline \multicolumn{3}{|l|}{ Faixa etária } \\
\hline 16 a 38 anos & 26 & 30,2 \\
\hline 39 a 59 anos & 31 & 36,1 \\
\hline 60 ou mais & 29 & 33,7 \\
\hline \multicolumn{3}{|l|}{ Sexo } \\
\hline Feminino & 49 & 56,9 \\
\hline Masculino & 37 & 43,1 \\
\hline \multicolumn{3}{|l|}{ Estado Conjugal } \\
\hline Solteiro (a) & 33 & 38,3 \\
\hline $\begin{array}{l}\text { Casado (a)/mora junto } \\
\text { com companheiro }(\mathrm{a})\end{array}$ & 46 & 53,4 \\
\hline Viúvo (a) & 7 & 8,3 \\
\hline
\end{tabular}


Em relação às condições de saúde dos usuários $76,7 \%$ possuíam problema crônico de saúde, com frequência maior entre os homens $(91,9 \%)$. Os principais problemas crônicos de saúde foram semelhantes entre homens e mulheres, as morbidades mais frequentes foram as DCV (59,2\% mulheres e $64,9 \%$ homens), neoplasias $(13,4 \%$ mulheres e $35,1 \%$ homens), e outros problemas $(10,2 \%$ mulheres e 10,8\% homens). Considerouse como outros problemas as doenças autoimunes (lúpus eritematoso sistêmico), leishmaniose, doenças genéticas (fibrose cística) entre outros (Tabela 2). A média de problemas crônicos de saúde por pessoa foi de 2,1 doenças.

A distribuição dos medicamentos utilizados também foi semelhante entre homens e mulheres, sendo os medicamentos mais utilizados os anti-hipertensivos $(36,7 \%$ mulheres e $45,9 \%$ homens), diuréticos (12,2\% mulheres e $12,2 \%$ homens), hipoglicemiantes $(8,1 \%$ mulheres $\mathrm{e}$ $13,5 \%$ homens). A média de medicamentos utilizados por pessoa foi de 3,6 (Tabela 2).

As principais causas de internação na UTI estavam relacionadas a complicações no pós-operatório ou a necessidade de monitoramento dos usuários no pós-operatório imediato $(37,9 \%)$ e insuficiência respiratória $(30,4 \%)$. Mulheres e homens tiveram causas de internação diferentes. Entre as mulheres, as causas de internação mais frequentes foram as complicações no pós-operatório imediato $(32,0 \%)$, choque séptico $(20,4 \%)$ e, insuficiência respiratória $(18,4 \%)$. Já entre os homens as causas foram choque séptico (51,3\%), pós-operatório $(47,8 \%)$ e, insuficiência respiratória $(32,4 \%)$ (Tabela 3).

Neste estudo $84,9 \%$ dos usuários estiveram hospitalizados por até 10 dias e $76,7 \%$ dos usuários tiveram alta da UTI para sua residência, ou transferência para outro hospital e/ou para enfermaria (Tabela 3).

0 percentual de morte entre os usuários foi de $23,3 \%$, com frequência maior entre os homens $(37,8 \%)$.

Tabela 2 - Distribuição das condições de saúde e utilização de medicações dos usuários internados na UTI. Cuiabá - MT, Brasil. 2016.

\begin{tabular}{|c|c|c|c|}
\hline Variável & $\begin{array}{l}\text { Fem } \\
(\%)\end{array}$ & $\begin{array}{c}\text { Mas } \\
(\%)\end{array}$ & $\begin{array}{c}\text { Total } \\
\text { (\%) }\end{array}$ \\
\hline \multicolumn{4}{|l|}{$\begin{array}{l}\text { Problema de saúde } \\
\text { crônico }\end{array}$} \\
\hline Sim & 65,3 & 91,9 & 76,7 \\
\hline Não & 34,7 & 8,1 & 23,3 \\
\hline \multicolumn{4}{|l|}{$\begin{array}{l}\text { Principais problemas } \\
\text { de saúde* }\end{array}$} \\
\hline $\begin{array}{l}\text { Doenças } \\
\text { cardiovasculares }\end{array}$ & 59,2 & 64,9 & 61,6 \\
\hline $\begin{array}{l}\text { Doenças do aparelho } \\
\text { respiratório }\end{array}$ & 4,1 & 16,2 & 9,3 \\
\hline Neoplasias & 13,4 & 35,1 & 25,6 \\
\hline Doenças hepáticas & 2,0 & 10,8 & 5,8 \\
\hline $\begin{array}{l}\text { Doenças do aparelho } \\
\text { excretor }\end{array}$ & 6,1 & 5,4 & 7,0 \\
\hline $\begin{array}{l}\text { Doenças do sistema } \\
\text { gastrointestinal }\end{array}$ & 0,0 & 10,8 & 4,6 \\
\hline SIDA & 2,0 & 5,4 & 3,5 \\
\hline $\begin{array}{l}\text { Outros } \\
\text { Principais } \\
\text { medicamentos } \\
\text { utilizados* }\end{array}$ & 10,2 & 10,8 & 10,5 \\
\hline Anti-hipertensivos & 36,7 & 45,9 & 40,7 \\
\hline Diuréticos & 12,2 & 12,2 & 13,9 \\
\hline Hipoglicemiantes & 8,1 & 13,5 & 10,4 \\
\hline $\begin{array}{l}\text { Antieméticos e anti- } \\
\text { ulcerosos }\end{array}$ & 4,1 & 10,8 & 7,0 \\
\hline Anticonvulsivantes & 6,1 & 2,7 & 4,6 \\
\hline Corticóides & 2,0 & 8,1 & 4,6 \\
\hline Antiplaquetários & 2,0 & 5,4 & 4,5 \\
\hline Antiinflamatórios & 2,0 & 5,4 & 4,5 \\
\hline Outros & 8,2 & 21,6 & 13,9 \\
\hline
\end{tabular}

*Variável de múltipla escolha. 
Tabela 3 - Distribuição das características da internação na UTI. Cuiabá - MT, Brasil. 2016.

\begin{tabular}{|c|c|c|c|}
\hline Variável & $\begin{array}{l}\text { Feminino } \\
(\%)\end{array}$ & $\begin{array}{l}\text { Masculino } \\
\text { (\%) }\end{array}$ & $\begin{array}{c}\text { Total } \\
(\%)\end{array}$ \\
\hline \multicolumn{4}{|c|}{$\begin{array}{l}\text { Unidade de } \\
\text { procedência }\end{array}$} \\
\hline $\begin{array}{l}\text { Unidades do } \\
\text { próprio } \\
\text { hospital }\end{array}$ & 77,5 & 86,5 & 81,4 \\
\hline $\begin{array}{l}\text { Hospital } \\
\text { público }\end{array}$ & 16,3 & 10,8 & 13,9 \\
\hline $\begin{array}{l}\text { Hospital } \\
\text { privado }\end{array}$ & 6,1 & 2,7 & 4,7 \\
\hline $\begin{array}{l}\text { Principais } \\
\text { causas de } \\
\text { internação* }\end{array}$ & & & \\
\hline Pós-operatório & 32,6 & 47,8 & 34,9 \\
\hline $\begin{array}{l}\text { Insuficiência } \\
\text { respiratória }\end{array}$ & 18,4 & 32,4 & 30,4 \\
\hline Choque séptico & 20,4 & 51,3 & 27,9 \\
\hline $\begin{array}{ll}\text { Problemas } & \\
\text { relacionados } & \text { a } \\
\text { gravidez } & \text { e } \\
\text { puerpério } & \end{array}$ & 12,2 & - & 7,0 \\
\hline $\begin{array}{l}\text { Emergência } \\
\text { cardiovascular }\end{array}$ & 10,2 & 2,7 & 7,0 \\
\hline Hemorragias & 6,1 & 2,7 & 4,7 \\
\hline $\begin{array}{l}\text { Insuficiência } \\
\text { Renal }\end{array}$ & 2,0 & 2,7 & 2,3 \\
\hline $\begin{array}{ll}\text { Outros } & \\
\text { Tempo } & \text { de } \\
\text { internação na } \\
\text { UTI }\end{array}$ & 12,2 & 13,5 & 12,7 \\
\hline Até 10 dias & 83,7 & 86,5 & 84,9 \\
\hline 11 a 20 dias & 8,2 & 8,1 & 8,1 \\
\hline 21 a 30 dias & 2,0 & 2,7 & 2,3 \\
\hline 31 dias ou mais & 6,1 & 2,7 & 4,6 \\
\hline $\begin{array}{l}\text { Desfecho } \\
\text { clínico }\end{array}$ & & & \\
\hline Alta da UTI & 87,8 & 62,2 & 76,7 \\
\hline Obito & 12,2 & 37,8 & 23,3 \\
\hline
\end{tabular}

*Variável de múltipla escolha.

As principais causas de morte na UTI entre as mulheres foram as pneumonias $(20,0 \%)$, disfunção de múltiplos órgãos $(15,0 \%)$ e choque séptico $(10,0 \%)$ (Figura 1$)$.

Já entre os homens, as principais causas de morte foram o choque séptico $(45,0 \%)$, disfunção de múltiplos órgãos $(40,0 \%)$ e pneumonia $(15,0 \%)$. Na categoria outros $(25,0 \%)$ foram incluídos trombose severa, acidose metabólica, leishmaniose, miocardiopatia, entre outras (Figura 1).

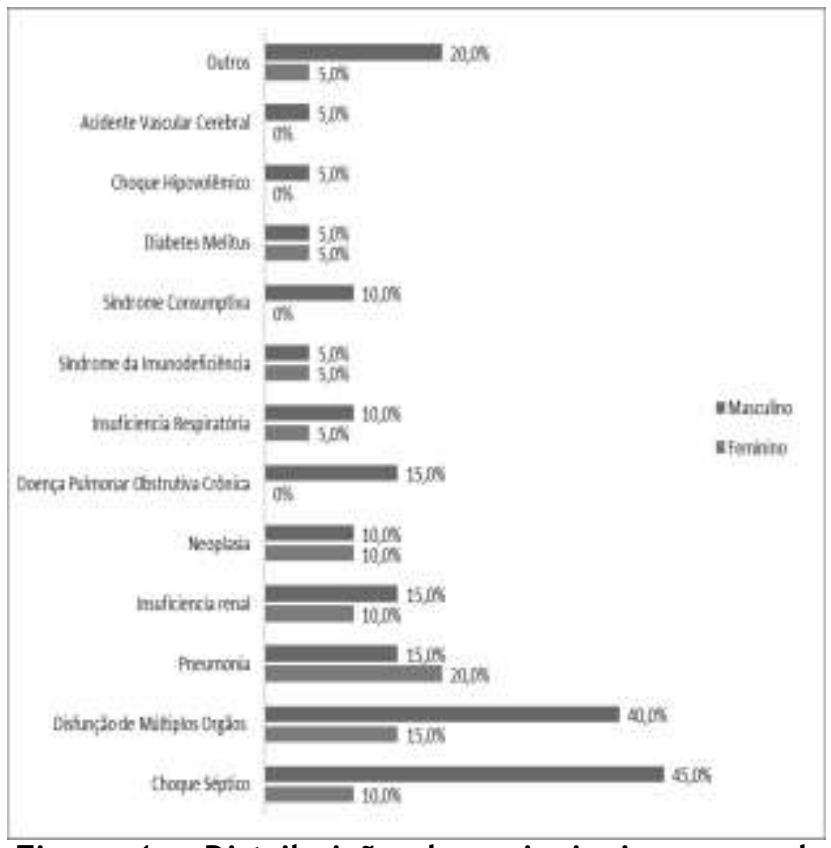

Figura 1 - Distribuição das principais causas de morte dos usuários internados na UTI. Cuiabá MT, Brasil. 2016.

\section{DISCUSSÃO}

Neste estudo houve a predominância de pessoas adultas, seguida do segmento de 60 anos ou mais, fato semelhante a outra investigação ${ }^{8}$. Em estudo realizado em uma UTI na Índia a mediana de idade de admissão foi de 53 anos 9 . Isso pode ser reflexo da recente transição demográfica e do aumento da expectativa de vida vivenciados nos países em desenvolvimento, como o Brasil $^{8}$.

Quanto ao estado conjugal, outro estudo com objetivo semelhante também encontrou predomínio de mulheres casadas 9 . 0 estado conjugal influencia diretamente a dinâmica familiar e 0 autocuidado, principalmente entre os idosos. Estudiosos têm apontado relação entre o estado civil e morbimortalidade, sendo maior a taxa de mortalidade em indivíduos viúvos, divorciados. Essa relação pode ser atribuída à influência familiar no cuidado à saúde, 
caracterizando o apoio do(a) companheiro(a) na procura por cuidado à saúde ${ }^{10}$.

As DCNT vendo sendo apontada em investigações como um problema importante ${ }^{5,11}$. Elas são responsáveis por $70,0 \%$ das mortes e estão relacionadas ao aumento do número de internações. Assim, o monitoramento destes fatores de risco pelos profissionais e serviços de saúde permite prevenir e controlar essas morbidades, evitando suas complicações ${ }^{5}$.

Outro fato constatado neste estudo e em outras investigações é a vulnerabilidade maior do sexo masculino às DCNT, geralmente eles possuem hábitos de vida menos saudáveis. Para o controle dessas doenças se faz necessário, o uso de medicamentos

contínuos, acompanhamento com profissionais de saúde, consultas periódicas e, principalmente hábitos de vida mais saudáveis ${ }^{8,11}$.

Visando legitimar direitos e ampliar as discussões acerca das fragilidades e dificuldades vivenciadas pela população masculina, surge em 2008, a Política Nacional de Atenção Integral à Saúde do Homem (PNAISH) ${ }^{12}$. No entanto, autores apontam motivos que ainda dificultam a efetivação desta política, principalmente a baixa procura dos homens na atenção primária de saúde, o que pode ser motivado por fatores como incompatibilidade de horário e questões de gênero ${ }^{8,13}$.

Nessa pesquisa a média de medicamentos utilizados pelos pacientes foi acima do número médio no Brasil $(2,4)^{14}$. Isso pode ocorrer devido ao aumento da prevalência das DCNT, maior acesso a aquisição de medicamentos e, a cultura que $o$ medicamento é a única conduta para o controle dos agravos 6 6-14,15.

0 maior acesso às medicações se deu por meio de iniciativas como a regulamentação dos genéricos (Lei 9.787, de 10 de Fevereiro de 1999), o Programa Farmácia Popular (Decreto 5.090 , de 20 de maio de 2004), o QualifarSUS (Portaria GM/MS 1.214), que garantem acesso gratuito aos medicamentos e/ou descontos de até $90 \%-14,15$.

As complicações do pósoperatório como uma das principais causas de internação na UTI, pode estar relacionada ao agravamento das condições de saúde das pessoas em geral, requerendo procedimentos invasivos e monitoramento das condições de saúde ${ }^{7}$.

Quanto ao tempo de internação, a maioria dos usuários ficou internada por até 10 dias, o que foi encontrado em pesquisas anteriores ${ }^{5-16}$. Esse achado pode ter ocorrido pela principal causa de internação encontrada neste estudo, pois pacientes em pós-operatório requerem menor período de internação. Sabe-se que no cuidado intensivo, a permanência do usuário é um indicador importante, pois pode influenciar significativamente em sua evolução. Quanto maior o período de internação na UTI, maior a probabilidade de infecções e ocorrência de eventos 
adversos que comprometem a segurança do paciente ${ }^{17,18}$.

Nesse estudo a frequência de óbito menor que o verificado em outras pesquisas $^{8}$. Investigação desenvolvida no Distrito Federal identificou frequência de óbito de $38,6 \%^{8}$. Outro estudo realizado no interior de Goiás verificou frequência de óbitos de $31,0 \%{ }^{19}$. O percentual de óbitos superior entre os homens pode ser explicado pela baixa adesão à prevenção de doenças, ocasionando a procura por serviços de saúde quando o problema está mais grave e com possíveis complicações ${ }^{7}$. Além disso, existem fragilidades na promoção à saúde do homem e no acesso aos serviços de saúde ${ }^{13}$.

A pneumonia foi a principal causa de morte entre as mulheres, semelhante ao encontrado em uma investigação anterior ${ }^{20}$. Para os homens chama a atenção a principal causa de morte, choque séptico, pois se trata de um problema grave, mais frequente nos homens em idades mais avançadas ${ }^{21}$. Nos Estados Unidos a sepse grave é uma das principais causas de morte em pacientes internados em UTI ${ }^{21}$. Assim, é imprescindível que medidas de segurança, como a identificação precoce da sepse, sejam estabelecidas e implementadas nas UTI.

\section{CONCLUSÃO}

A maioria dos usuários internados na UTI era mulher, na faixa etária de 39 a 59 anos, casada ou residia com 0 companheiro. Os principais problemas crônicos e medicamentos utilizados foram semelhantes entre homens e mulheres.

As principais causas de internação entre as mulheres foram pós-operatório imediato, choque séptico e insuficiência respiratória, enquanto para os homens foram choque séptico, pós-operatório e insuficiência respiratória. Grande parte dos usuários ficou internada por até 10 dias e 23,3\% morreram, com maior frequência entre os homens.

Constatou-se que as principais causas de morte na UTI entre as mulheres foram pneumonia, disfunção de múltiplos órgãos e choque séptico. Para os homens foram o choque séptico, disfunção de múltiplos órgãos e pneumonia.

As internações na UTI trazem impacto não somente para os serviços de saúde, mas, sobretudo para a família e pessoa que a vivencia.

Embora seja um estudo descritivo, esse perfil de investigação ainda é escasso na região do estudo. Os achados dessa pesquisa mostram-se importantes para a gestão de recursos e planejamento da assistência, não somente na atenção terciária, mas também permitem pensar intervenções no âmbito da atenção primária, com objetivo de prevenir complicações das DCNT.

\section{REFERÊNCIAS}

1. Souza MFM, Malta DC, França EB, Barreto LM. Changes in health and disease in Brazil and its States in the 30 years since the 
Unified Healthcare System (SUS) was created. Ciênc Saúde Colet. 2018; 23(6):1737-1750.

2. Wilkins $E$, Wilson $L$, Wickramasinghe $\mathrm{K}$, Bhatnagar $\mathrm{P}$, Leal J, Luengo-Fernandez R, et al. European Cardiovascular Disease Statistics 2017. European Heart Network, Brussels [internet]. 2017 [acesso em 2019 Jul 16]: 192 p. Disponível em: http://www. ehnheart.org/image s/CVD-statistics-report-August2017.pdf

3. Ministério da Saúde (BR). Secretaria de Vigilância em Saúde. Departamento de Vigilância de Doenças e Agravos não Transmissíveis e Promoção da Saúde. Relatório do III Fórum de Monitoramento do Plano de Ações Estratégicas para o Enfrentamento das Doenças Crônicas não Transmissíveis no Brasil. Brasília: Ministério da Saúde, 2018. 121 p.

4. Ministério da Saúde (BR). Secretaria de Vigilância em Saúde. Departamento de Vigilância de Doenças e Agravos Não Transmissíveis e Promoção da Saúde. Saúde Brasil Estados 2018: uma análise de situação de saúde segundo o perfil de mortalidade dos estados brasileiros e do Distrito Federal. Brasília: Ministério da Saúde, 2018. 374 p.

5. Melo ACL, Menegueti MG, Laus AM. Profile of patients in intensive care: considerations for the nursing team. Rev enferm UFPE on line. 2014; 8(9):3142-8.

6. Ribeiro AL, Duncan BB, Brant LC, Lotufo PA, Moinho JG, Barreto $S M$. Cardiovascular Health in Brazil: Trends and Perspectives. Circulation. 2016; 133(4): 42233.

7. Rodriguez $A H$, Bub $M B C$, Perão OF, Zandonadi G, Rodriguez MJH. Epidemiological characteristics and causes of deaths in hospitalized patients under intensive care. Rev Bras Enferm. 2016; 69(2):229-34.
8. Guia CM, Biondi RS, Sotero $S$, Lima AA, Almeida KJQ, Amorim FF. Epidemiological profile and predictors of mortality in an intensive care unit in a general hospital in Distrito Federal. Comun Ciênc Saúde. 2015; 26(1/2): 9-19.

9. Gudivada KK, Krishna B, Sriram S. Evaluation of quality indicators in an Indian intensive care unit using CHITRA database. Indian J Crit Care Med. 2017; 21(12):841846.

10. Levorato $C D$, Mello $L M$ de, Silva AS, Nunes AA. Factors associated with the demand for health services from a gender-relational perspective. Ciênc Saúde Colet. 2014; 19(4):1263-74.

11. Garland A, Olafson K, Ramsey $C D$, Yogendranc $M$, Fransoo $R$. Reassessing access to intensive care using an estimate of the population incidence of critical illness. Critical Care. 2018; 22(1):208.

12. Ministério da Saúde (BR). Secretaria de Atenção à Saúde. Departamento de Ações Programáticas Estratégicas. Política Nacional de Atenção Integral à Saúde do Homem: princípios e diretrizes. Brasília: Ministério da Saúde; 2008.

13. Prado MAMB, Francisco PMSB, Bastos TF, Barros MBA. Use of prescription drugs and selfmedication among men. Rev Bras Epidemiol. 2016; 19(3): 594608.

14. Lima MG, Álvares J, Guerra Júnior AA, Costa EA, Guibu IA, Soeiro $O M$, et al. Indicadores relacionados ao uso racional de medicamentos e seus fatores associados. Rev Saúde Pública. 2017; 51(Supl 2):23s.

15. Bertoldi AD, Dal Pizzol TS, Ramos LR, Mengue SS, Luiza VL, Tavares NUL. Perfil sociodemográfico dos usuários de medicamentos no Brasil: resultados da PNAUM 2014. Rev Saúde Pública. 2016; 50(supl 2):1-11.

16. Sulieman $\mathrm{H}$, El-Mahdi $\mathrm{W}$, Awadekareem M, Nazer $L$. 
Characteristics of Critically-Ill Patients at Two Tertiary Care Hospitals in Sudan. Sultan Qaboos Univ Med J. 2018; 18(2):e190e195.

17. Barros LLS, Maia CFS, Monteiro MC. Risk factors associated to sepsis severity in patients in the Intensive Care Unit. Cad Saúde Colet. 2016; 24(4):388-396.

18. Ball IM, Bagshaw SM, Burns KE, Cook DJ, Day AG, Dodek PM, et al. A clinical prediction tool for hospital mortality in critically ill elderly patients. J Crit Care. 2016; 35:206-12.
19. Castro RR, Barbosa NB, Alves T, Najberg E. Profile of hospitalization in intensive care units for adults in Anápolis, Goiás - 2012. Rev gest sist saúde. 2016; 5 (2).

20. Silva GM, Menezes GGS. Evaluation of socio demographic profile and lifestyle of hospitalized patients in the city of Lagarto, Sergipe. Scientia Plena. 2014; 10(3):1-9.

21. Mayr FB, Yende $S$, Angus DC. Epidemiology of severe sepsis. Virulence. 2014; 5(1):4-11.

Conflito de interesses: Os autores declaram não haver conflito de interesses.

Participação dos autores:

- Concepção: Cruz YV, Cardoso JDC.

- Desenvolvimento: Cruz YV, Cardoso JDC.

- Redação e revisão: Cruz YV, Cardoso JDC, Cunha CRT, Vechia ADRD.

Como citar este artigo: Cruz YV, Cardoso JDC, Cunha CRT, Vechia ADRD. Perfil da morbimortalidade da unidade de terapia intensiva de um hospital universitário. J Health NPEPS. 2019; 4(2):230-239. 